\title{
Overshadowing as a function of trial number: Dynamics of first- and second-order comparator effects
}

\author{
STEVEN STOUT, FRANCISCO ARCEDIANO, MARTHA ESCOBAR, and RALPH R. MILLER \\ State University of New York, Binghamton, New York
}

\begin{abstract}
In two conditioned lick suppression experiments with rats, we examined the permanence of the overshadowing effect as a function of the number of compound reinforced training trials. In Experiment 1, robust overshadowing was observed following 4 compound-US pairings but dissipated with 36 pairings. Overshadowing decreased because responding to the overshadowed stimulus increased, not because responding by the control group decreased. This dissipation was stimulus specific and not attributable to a response ceiling. Experiment 2 extended the generality of the effect to a sensory preconditioning design and further demonstrated that overshadowing lost through many compound-US pairings was restored by posttraining extinction of the training context. The results are explicable in terms of the extended comparator hypothesis (Denniston, Savastano, \& Miller, 2001) under the assumption that the impacts of first- and second-order comparator processes grow differentially as a function of number of trials.
\end{abstract}

Overshadowing refers to the common observation that conditioned responding elicited by a target stimulus $(\mathrm{X})$ is often lower after it has been reinforced in compound with a (usually) more salient stimulus (A; i.e., AX $\rightarrow$ outcome) than after it has been reinforced alone $(\mathrm{X} \rightarrow$ outcome). Overshadowing occurs within many conditioning preparations, including salivary conditioning (Pavlov, 1927), rabbit nictitating membrane conditioning (e.g., Kehoe, 1982), conditioned lick suppression (e.g., Blaisdell, Denniston, \& Miller, 1998), and human contingency judgment (Price \& Yates, 1993). The ability to account for overshadowing, as representative of cue competition, has become a benchmark for contemporary models of acquired behavior. Most theories of conditioned responding explain overshadowing by postulating that cues that are concurrently present during pairings with the unconditioned stimulus (US) compete for some limited resource (such as associative strength or attention). The amount of this resource controlled by a cue determines its response potential. Most influential among these limited-resource models of overshadowing is the theory of Rescorla and Wagner (1972), according to which a given US is capable of supporting a finite amount of associative strength

Support for this research was provided by NIMH Grants 33881 and 24690. We thank Jeffrey Amundson, Raymond Chang, and Daniel Wheeler for their insightful advice concerning all aspects of this research. Correspondence concerning this article should be addressed to R. R. Miller, Department of Psychology, SUNY-Binghamton, Binghamton, NY 13902-6000 (e-mail: rmiller@binghamton.edu).

-Accepted by the previous editorial team of Ralph R. Miller. Vincent M. LoLordo was the action editor for this manuscript. on any given trial. Associative strength is ordinarily partitioned among cues presented together during a trial according to their relative salience. Specifically, the model states that on a given trial, $\Delta V_{i}=\alpha_{\mathrm{i}} \beta\left(\lambda-\Sigma V_{i}\right)$, where $\Delta V_{i}$ is the change in associative strength, $V_{i}$, of the $i$ th cue, $\lambda$ is the total associative strength supportable by the US, and $\Sigma V_{i}$ is the sum of the associative strength of all cues present on the trial. $\beta$ represents a learning rate parameter determined by the salience of the US. Critical to the analysis of overshadowing is the cue-specific learning rate parameter, $\alpha_{i}$, which increases with the salience of the $i$ th cue. According to the model, the change in associative strength of cues conditioned in compound is directly proportional to the difference between $\lambda$ and their summed associative strengths. Conditioned responding to each cue approaches an asymptote as the sum of the associative strengths of the overshadowing and overshadowed cues approaches $\lambda$. Because the rates at which the associative strengths of two compounded cues (e.g., $A$ and $X$ ) increase are directly proportional to their salience, at asymptote the ratio of the associative strengths of $\mathrm{A}$ and $\mathrm{X}$ (i.e., $V_{\mathrm{A}}: V_{\mathrm{X}}$ ) will be equal to the ratio of their saliences (i.e., $\alpha_{\mathrm{A}}: \alpha_{\mathrm{X}}$ ). Rescorla and Wagner did not specify a response rule by which associative strength is translated into performance, other than to say that the relationship is monotonic. The basic assumption that overshadowing reflects a competition for some limited resource (and thus should be permanent provided there is no further training with the target stimulus) is shared by many other models of Pavlovian conditioning, including the models offered by Gallistel and Gibbon (2000), McLaren and Mackintosh (2000), Pearce and Hall (1980), and Van Hamme and Wasserman (1994). 
At least two contemporary models of conditioning do not view overshadowing as resulting from a competition for any finite resource. Mackintosh (1975) criticized the limited-resource view of compound conditioning and proposed, instead, his own modification of Bush and Mostellar's (1951) linear operator account of the effects of acquisition and extinction. Mackintosh's model of compound conditioning assumes that changes in the learning rate parameter, $\alpha$, denoting attention, are driven by a comparison of the relative associative strengths of the two cues (overshadowing and overshadowed). The value of $\alpha$ for the cue that best predicts the outcome increases, whereas the $\alpha$ s of all cues that are weaker predictors of the outcome decrease. Because the initial value of $\alpha$ is higher for the most salient cue, after a single conditioning trial its associative strength will also exceed that of less salient cues. As a result of the comparison process, the $\alpha$ value for the overshadowed (i.e., less salient) cue will decline. Importantly, in Mackintosh's model, as in Bush and Mostellar's theory, the potential asymptotic associative strength for each cue is unaffected by the other cue's presence. Mackintosh's model can therefore accommodate any decline in overshadowing seen following extended training on the simple assumption that the less salient element of the compound continues to condition, albeit more slowly than the more salient element and more slowly than an equally salient cue conditioned alone. Nevertheless, in Mackintosh's model, the rate at which $\alpha$ for the less salient cue declines is not precisely specified. If this rate of decline is very rapid and the salience of the overshadowed cue approaches zero before the cue's associative strength reaches $\lambda$, the associative strength of the overshadowed cue will reach an asymptote at a level that is lower than $\lambda$. Thus, the model predicts that overshadowing should fade with extended training, although this prediction is parameter dependent and is not a categorical feature of the model.
Like Mackintosh's (1975) model, the comparator hypothesis of Miller and Matzel (1988) proposes that cues become associated with the US independently of each other despite being trained in compound. Responding to the target conditioned stimulus (CS) (X) is directly related to the strength of the X-US association (called Link 1; see Figure 1) and is inversely related to the product of the strengths of the $\mathrm{X}-\mathrm{A}$ association (Link 2) and the A-US association (Link 3). That is, conditioned responding is attenuated to the degree that CS X is indirectly linked to the US through the overshadowing stimulus (A). The stimuli through which the target CS is indirectly associated with the US are called the comparator stimuli. In simple conditioning (i.e., $\mathrm{X} \rightarrow \mathrm{US}$ ), the only comparator stimulus for the CS is the conditioning context; but in the case of overshadowing (i.e., $\mathrm{AX} \rightarrow \mathrm{US}$ ), the principle comparator stimulus is the overshadowing cue. Support for the comparator interpretation of overshadowing comes from experiments in which the overshadowing stimulus (A) is presented alone (extinction) following overshadowing training and prior to testing with CS X. According to the comparator hypothesis, these extinction trials should weaken Links 2 and 3 and, hence, the net overshadowing effect. Consistent with this prediction, several investigators (e.g., Cole, Oberling, \& Miller, 1999; Kaufman \& Bolles, 1981; Matzel, Schachtman, \& Miller, 1985; Miller, Barnet, \& Grahame, 1992) have found that posttraining extinction of the more salient (overshadowing) CS restored robust conditioned responding to the less salient (overshadowed) element of the stimulus compound (but see Holland, 1999; Rauhut, McPhee, \& Ayres, 1998). According to the comparator hypothesis's account of overshadowing, if the associative strengths of Links 2 and 3 increase over reinforced trials according to a monotonic function, overshadowing should become more pronounced with extended compound training. Thus, the comparator hy-

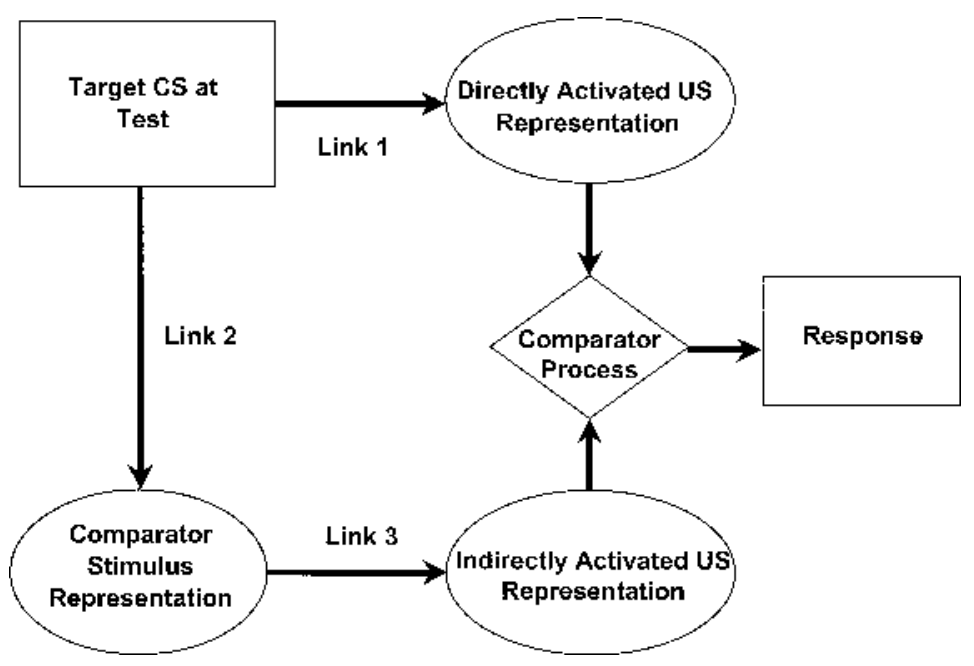

Figure 1. The original comparator hypothesis (Miller \& Matzel, 1988). 
pothesis predicts that overshadowing should increase with number of compound training trials.

An opposite view of the role of a target CS's indirect association with the US is that it enhances, rather than suppresses, excitatory responding. If this occurred in an overshadowing paradigm, then following extended training, an overshadowed cue would come to elicit a type of second-order conditioning in which, at test, the target stimulus would activate a representation of the US mediated by the representation of the overshadowing stimulus. If this indirectly activated US representation summed with the directly activated US representation, one would expect to observe attenuated overshadowing or, perhaps, even potentiation. Several investigators have shown that, under certain conditions, compound reinforcement can potentiate conditioned responding relative to an elementally reinforced control condition (e.g., Rusiniak, Hankins, Garcia, \& Brett, 1979). Durlach and Rescorla (1980) observed that posttraining extinction of the potentiating element reduced responding to the target element. Presumably, this manipulation extinguished the within-compound association and, thus, reduced the target CS's potential to indirectly retrieve the US representation. Of course, there is the basic question of under what conditions posttraining extinction of the associate element increases responding to the target element, as is predicted by the comparator hy pothesis, or reduces it, as is observed in the potentiation paradigm. The answer is not yet entirely clear (for a discussion of this mediation paradox, see Miller \& Escobar, 2002).

Toward better understanding the basis of cue competition in general and particularly overshadowing, the present research tested the permanence of overshadowing and the effect of contextual extinction. To summarize, a waning of overshadowing with extended compound trials would clearly raise problems for most contemporary accounts of overshadowing, which anticipate extended training will yield asymptotic overshadowing (e.g., Miller \& Matzel, 1988; Rescorla \& Wagner, 1972). Thus, with the parametrically qualified exception of Mackintosh's (1975) theory and Durlach and Rescorla's (1980) within-compound view of potentiation, contemporary theoretical accounts of Pavlovian conditioning predict that overshadowing should not wane with additional conditioning trials.

Although the permanence of overshadowing over extended compound training trials does not appear to have been explicitly tested by experimental analysis, at least two studies may be relevant to this issue. Bellingham and Gillette (1981), using a water-reinforced conditioned magazine entry preparation with rats, reported that responding to the separate elements of a compound CS increased with the number of compound reinforced trials. Conditioned responding initially increased more rapidly to the more salient element than to the less salient element of the compound, but this difference in responding waned with extended training as responding to the less salient element increased to the same level as responding to the more salient element. However, their study cannot be taken as an unequivocal demonstration that overshadowing dissipates with extended training, because there was no control group trained and tested with the less salient stimulus alone (i.e., there was no appropriate control for overshadowing).

Azorlosa and Cicala (1988), using a conditioned lick suppression procedure with rats, observed that the closely related phenomenon of blocking wanes with additional $\mathrm{AX} \rightarrow \mathrm{US}$ pairings. Blocking differs from overshadowing in that the two stimuli are typically of similar salience and CS A is made either more salient or a better predictor of the US by its being reinforced alone prior to its being paired with CS X (Kamin, 1969). In Azorlosa and Cicala's study, suppression to the target (blocked) CS (X) increased to the level of a control stimulus (also conditioned in compound, but without prior $\mathrm{A} \rightarrow \mathrm{US}$ pairings) within 10 compound trials, despite blocking having been observed following 1 compound trial. A procedural limitation of their between-subjects study was that it confounded the number of $\mathrm{AX} \rightarrow \mathrm{US}$ pairings with the number of US presentations. Thus, it is possible that blocking declined (i.e., conditioned responding to $X$ increased) because of the number of times the US was presented in the experimental context (i.e., pseudoconditioning of $\mathrm{X}$ ), rather than the number of times the AX compound was paired with the US.

The purpose of Experiment 1 was to examine whether overshadowing dissipates with extended AX $\rightarrow$ US pairings. The experiment avoided the design limitations of Azorlosa and Cicala's (1988) and Bellingham and Gillette's (1981) studies by (1) using a control condition in which the less salient (overshadowed) element was reinforced alone, (2) testing for the stimulus specificity of any observed dissipation of overshadowing, and (3) equating all subjects on the number of US presentations.

\section{EXPERIMENT 1}

In the first experiment, we examined whether overshadowing dissipates as the number of reinforced pairings of the compound cue with the US increases. The experiment used a mixed design (i.e., between- and withinsubjects variables) that equated the number of $\mathrm{CS} \rightarrow \mathrm{US}$ pairings in each group (see Table 1). Each subject received independent pairings of a shock US with each of two low-salience target stimuli (X and Y). CS X was reinforced 36 times, whereas CS Y was reinforced 4 times. We manipulated whether CS X or CS Y was accompanied by a more salient potentially overshadowing stimulus, A or B, respectively, which created a 2 (treatment of $\mathrm{X}[\mathrm{X}$ vs. AX]) $\times 2$ (treatment of Y [Y vs. BY]) factorial design. Thus, all the subjects received 40 shocks, and any loss of overshadowing observed with CS X relative to CS Y could not be explained by differences in stimulusnonspecific fear caused by different numbers of footshocks (i.e., pseudoconditioning). In addition to testing CSs X and Y, A and B were tested to see whether re- 
Table 1

Experiment 1 Design Summary

\begin{tabular}{llll}
\hline \multicolumn{1}{c}{ Group } & Phase 1 in Cxt2 & \multicolumn{1}{c}{ Phase 2 in Cxt2 } & \multicolumn{1}{c}{ Test in Cxt1 } \\
\hline Comp/Comp & A-, B-, X-, Y- & AX $\rightarrow$ US, BY $\rightarrow$ US & X or A, Y or B \\
Comp/Ele & A-, B-, X-, Y- & AX $\rightarrow$ US, Y $\rightarrow$ US & X or A, Y or BY \\
Ele/Comp & A-, B-, X-, Y- & X $\rightarrow$ US, BY $\rightarrow$ US & X, Y or B \\
Ele/Ele & A-, B-, X-, Y - & X $\rightarrow$ US, Y $\rightarrow$ US & X, Y or BY \\
\hline
\end{tabular}

Notes-Comp, compound; Ele, element; Cxt1, Context 1; Cxt2, Context 2; X, low salience target stimulus reinforced 36 times in Phase 2; Y, low salience target stimulus reinforced 4 times in Phase 2; A, high salience (overshadowing) stimulus for X; B, high salience (overshadowing) stimulus for Y; US, footshock; -, nonreinforced presentations; $\rightarrow$, followed by.

sponding to the more salient stimuli was asymptotic after 4 BY-US pairings (relative to $36 \mathrm{AX}$-US pairings). In addition, to determine whether the overshadowing of $Y$ after 4 pairings was due to cue competition or stimulus generalization decrement (as a result of training of BY and testing on $\mathrm{Y}$ ), some subjects were trained with $\mathrm{Y}$ and tested on BY. This control for generalization decrement was predicated on Pearce's (1987) argument that generalization from BY to $Y$ should be equivalent to that from Y to BY (but see Brandon, Vogel, \& Wagner, 2000).

\section{Method}

\section{Subjects}

The subjects were 24 male and 24 female Sprague Dawley rats, bred in our colony. They were individually housed in wire-mesh cages in a vivarium maintained on a 16:8-h light:dark cycle. Running occurred approximately midway through the light portion of the cycle. A progressive water deprivation schedule was imposed over the week prior to the beginning of the experiment until water availability was limited to $30 \mathrm{~min}$ per day, available roughly $1 \mathrm{~h}$ after any experimental manipulation. The subjects were assigned to four groups of $n=12$ : Comp/Comp, Comp/Ele, Ele/Comp, and Ele/Ele, counterbalanced for sex. With Comp denoting compound training and Ele denoting elemental training, the first term of the group name designates treatment of CS X, which was presented and reinforced 36 times, whereas the second term designates the treatment of CS Y, which was presented and reinforced 4 times.

\section{Apparatus}

Six identical copies of each of two different types of experimental chambers were used. Chamber Rectangular $(\mathrm{R})$ was a clear Plexiglas rectilinear chamber, measuring $23.0 \times 8.5 \times 12.5 \mathrm{~cm}$ (length $\times$ width $\times$ height). The floor was constructed of 0.48 -cm-diameter stainless steel rods, spaced $1.5 \mathrm{~cm}$ apart, center to center. The rods were connected by NE- 2 neon bulbs that allowed a constant-current footshock to be delivered by means of a 5,000-V AC circuit in series with a $1.0-\mathrm{M} \Omega$ resistor. Each copy of Chamber $\mathrm{R}$ was housed in a separate light- and sound-attenuating environmental isolation chest, which was dimly illuminated by a $2-\mathrm{W}$ (nominal at 120 VAC) incandescent bulb driven at $60 \mathrm{VAC}$. The houselight was mounted on the ceiling of the environmental chest, approximately $26 \mathrm{~cm}$ from the center of the experimental chamber.

Chamber V-shaped (V) was a 22.1-cm-long box in a truncated$\mathrm{V}$ shape $(25.3-\mathrm{cm}$ height, $21.3 \mathrm{~cm}$ wide at the top, $5.1 \mathrm{~cm}$ wide at the bottom). The floor and sides were constructed of stainless steel sheets, and the ceiling was constructed of clear Plexiglas. The floor of each chamber consisted of two parallel metal plates, each $2.0 \mathrm{~cm}$ wide, with a $1.1-\mathrm{cm}$ gap between them. Each V-shaped chamber was housed in its own environmental isolation chest, which was dimly illuminated by a 7.5-W (nominal at 120 VAC) incandescent houselight driven at $60 \mathrm{VAC}$, mounted on an inside wall of the environmental chest, approximately $30 \mathrm{~cm}$ from the center of the experimental chamber. The light entering the animal chamber was primarily that reflected from the roof of the environmental chest, which was made of white sound-insulating tiles. The light intensities in the two types of chambers were approximately equal, due to the differences in opaqueness of the walls in Chambers $\mathrm{R}$ and $\mathrm{V}$. Chambers $\mathrm{R}$ and $\mathrm{V}$ were counterbalanced within groups in serving as Contexts 1 and 2, respectively. Training occurred in Context 2, whereas all other phases of the experiment occurred in Context 1. This use of separate contexts for training and testing assured that testing occurred in a relatively neutral context.

Each chamber ( $\mathrm{R}$ and $\mathrm{V}$ ) could be equipped with a water-filled lick tube that extended $1 \mathrm{~cm}$ from the rear of a cylindrical niche, $4.5 \mathrm{~cm}$ in diameter, left-right centered in one short wall, with its axis perpendicular to the wall, and positioned with its center $4.25 \mathrm{~cm}$ above the floor of the chamber. Each niche had a horizontal infrared photobeam traversing it parallel to the wall on which the niche was mounted, $1 \mathrm{~cm}$ in front of the lick tube. In order to drink from the tube, the subjects had to insert their heads into the niche, thereby breaking the infrared photobeam. Thus, we could record when the subjects had their heads in the niche. Ordinarily, they did this only when they were drinking. Disruption of ongoing drinking by a test stimulus served as our dependent variable.

Each chamber ( $\mathrm{R}$ and $\mathrm{V}$ ) was also equipped with three speakers widely separated on the inside walls of the environmental chest. Each speaker could deliver a different auditory stimulus. These were a complex tone composed of two low frequencies (300 and $340 \mathrm{~Hz}$ ) presented simultaneously, a click train $(6 / \mathrm{sec})$, and a white noise. In addition, a relay could provide a buzzing sound. Ventilation fans in each environmental chest provided an average 74- $\mathrm{dB}(\mathrm{C})$ background noise. The tone and buzzer $(12 \mathrm{~dB}[\mathrm{C}]$ above background) served as the potentially overshadowing stimuli (A and B, counterbalanced). The clicks and white noise $(6 \mathrm{~dB}[\mathrm{C}]$ above background) served as the potentially overshadowed stimuli (X and Y, counterbalanced). The US consisted of a 0.5 -sec, 1.0-mA footshock.

\section{Procedure (See Table 1)}

Acclimation. On Day 1, all the subjects were exposed to Context 1 for $60 \mathrm{~min}$, during which time the lick tubes were present. The purpose of pretraining exposure was to allow the subjects to locate and adapt to the lick tubes. No nominal stimuli were presented.

Phase 1 (stimulus preexposure). During a 60 -min session in Context 2, each of the four stimuli were presented twice to all the animals in the following sequence: tone, click, buzzer, noise, noise, buzzer, click, and tone, with a 7-min intertrial interval. Each stimulus presentation lasted for $5 \mathrm{sec}$. The purpose of stimulus pretraining exposure was to habituate unconditioned responding to the stimuli and to decrease the possibility of configural conditioning during the compound training. This nonreinforced stimulus preexposure 
was conducted in the training context rather than in the testing context to prevent the test context from possibly becoming a negative occasion setter (i.e., a signal that the CSs would not be reinforced).

Phase 2 (training). During Days 3-12, all the subjects received $4 \mathrm{CS} \rightarrow$ US pairings per daily 60-min session in Context 2 . CSs were presented according to two schedules in alternation. On Schedule 1, CSs occurred at 7, 18, 41, and 52 min into the session. On Schedule 2, CSs occurred at 5,23, 32, and $50 \mathrm{~min}$ into the session. Of the $40 \mathrm{CS} \rightarrow$ US pairings, 36 were with CS X, and 4 were with CS Y. The $4 \mathrm{Y} \rightarrow$ US pairings were scheduled as 1 daily pairing on each of Days 3, 6, 9, and 12 of the experiment. Over these days, the serial position of the $\mathrm{Y} \rightarrow \mathrm{US}$ pairing was varied in the following sequence: $1,4,3$, and 2 . This was done to equate CSs $\mathrm{X}$ and $\mathrm{Y}$ on the average position they occupied during Phase 2. When CSs A and B occurred (see Table 1), these stimuli were simultaneous with CSs X and Y. All the stimuli were $5 \mathrm{sec}$ in duration. The footshock US occurred during the last $0.5 \mathrm{sec}$ of CS presentation, making CS and US termination simultaneous.

Reacclimation. On Days 13-15, all the subjects were reexposed to Context 1 during daily 60 -min sessions in order to restabilize baseline drinking, which is ordinarily disrupted by the footshock US. Lick tubes were available, and no nominal stimuli were presented. Times to complete the first five cumulative seconds of licking were recorded. By Day 15, all the subjects drank from the lick tubes for five cumulative seconds within $60 \mathrm{sec}$ of placement into the test apparatus.

Testing. Testing took place in Context 1 on Days 16 and 17. Fear of $\mathrm{X}$ and $\mathrm{Y}$, as indexed by their potential to suppress ongoing drinking, was assessed by presenting the test stimulus immediately after completion of the first five cumulative seconds of licking on each day, thereby ensuring that all the subjects were licking at the time of CS onset. Subjects failing to drink for at least five cumulative seconds within $60 \mathrm{sec}$ of placement into the chamber were scheduled to be eliminated from the study, owing to their exhibiting unusually high generalized fear of the test context. Times from placement into the box until the first five cumulative seconds of drinking (pre-CS time) were analyzed to ensure that there were no appreciable differences among groups. However, the primary dependent measure was the time following CS onset that it took for each sub- ject to complete an additional five cumulative seconds of licking (CS time). The CS remained on for $15 \mathrm{~min}$. Thus, the CS time variable was limited to a maximum of $15 \mathrm{~min}$. To improve the normality of the within-group pre-CS - and CS-time distributions, pre-CS and CS times were converted to logarithms (base 10) for the statistical analyses. An $\alpha$ value of .05 was preselected as the criterion for statistical significance.

The scheme used to determine the stimulus tested on each day of testing is summarized in Table 1. For the first day of testing (Day 16), the stimuli tested in each group were as follows. In Group Comp/Comp, 3 subjects each were tested with A, B, X, or Y. In Group Comp/Ele, 3 subjects each were tested with A, BY, X, or Y. In Group Ele/Comp, 6 subjects were tested with $X$, and 3 each with $\mathrm{Y}$ or B. In Group Ele/Ele, 6 subjects were tested with X, and 3 each with Y or BY.

The second day of testing (Day 17) followed the same scheme as the 1st day, with the provision that each subject was tested with the other target cue. On each day of testing, all the stimuli were counterbalanced on the variables of physical identity of the stimulus, sex of the subject, and type of training context (i.e., R or V).

\section{Results and Discussion}

The results are presented by group in Figure 2 and by condition in Figure 3. As can be seen, CS Y, which was reinforced 4 times, elicited less responding if it had been reinforced in compound with a more salient stimulus (CS B) than if it had not (compound training vs. elemental training, respectively). By contrast, CS X, which was reinforced 36 times, was unaffected by its having been reinforced in the presence of a more salient cue (CS A). Responding to both overshadowing cues (A and B; not depicted, see means below) was approximately the same. A comparison between the responding elicited by CS A and CS B suggests that there was little, if any, effect of additional reinforced training on A relative to B. Statistical analyses confirmed these conclusions. One subject

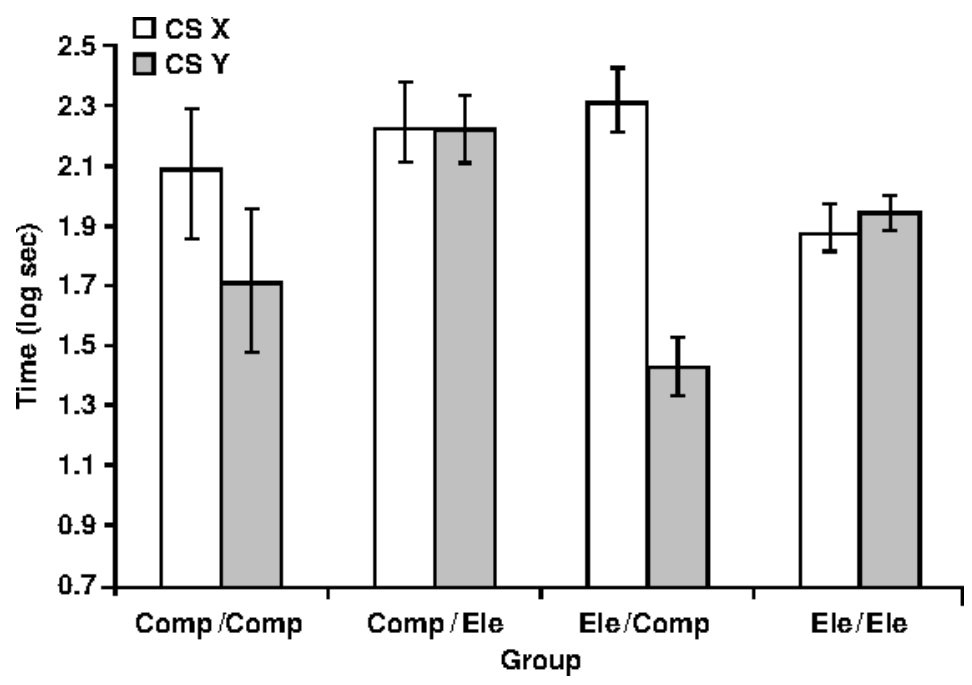

Figure 2. Mean times to resume drinking upon presentation of the test stimulus in Experiment 1. During training, conditioned stimuli (CSs) $X$ (white bars) and $Y$ (gray bars) were reinforced in compound with a more salient stimulus (Comp) or alone (Ele). CS X was reinforced 36 times, whereas CS Y was reinforced 4 times, over the course of the acquisition phase. Error bars indicate standard errors of the means. 


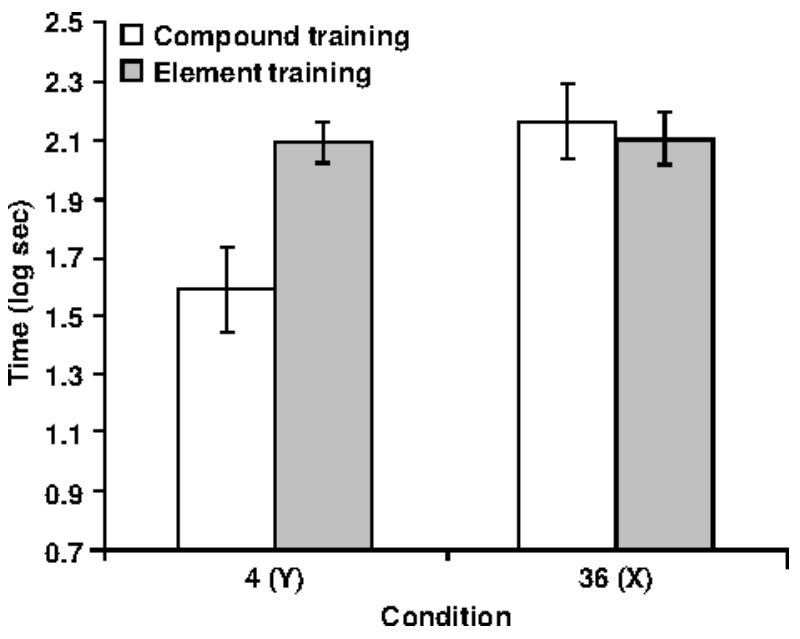

Figure 3. Mean times to resume drinking upon presentation of the test stimulus in Experiment 1, by condition (collapsed across groups).

from Group Comp/Ele was excluded, owing to a pre-CS time greater than $60 \mathrm{sec}$.

A first factorial (compound vs. element, X vs. Y) analysis of variance (ANOVA) tested whether pre-CS times differed across conditions. They did not ( $p \mathrm{~s}>.76)$. Thus, baseline levels of fear were approximately equal across conditions. This would seem to preclude an explanation of the seeming dissipation of overshadowing as resulting from the summation of generalized contextual excitation with the excitation elicited by the test cue.

The remaining analyses were of lick suppression times in the presence of the test CS. An initial issue was whether the overshadowing apparently observed with CS Y could be attributed to generalization decrement produced by testing with a different stimulus combination from the one reinforced during acquisition (i.e., BY). A between-subjects comparison of suppression to CS Y alone with suppression to B and $\mathrm{Y}$ in compound for the subjects trained on $\mathrm{Y}$ alone addressed this possibility. Following Pearce (1987), our assumption was that generalization decrement from $\mathrm{Y}$ to BY should be equal to the decrement from BY to Y. After training with CS Y alone, whether it was presented alone $(M=2.09 \log \mathrm{sec})$ or in compound ( $M=2.10 \log \mathrm{sec})$ with Stimulus B at test did not significantly affect conditioned responding $(p>.90)$.

The next analysis addressed whether responding to a test stimulus (X, Y, A, or B) depended on the order of the test session. This question was addressed with a test session (1 vs. 2$) \times$ test stimulus $(\mathrm{X}$ [or A] vs. Y [or BY or B] tested first) ANOVA. Because this design was not a complete factorial, test stimulus was treated as an independent factor, rather than as a repeated measures factor. Owing to the resultant larger number of degrees of freedom, this constituted a bias toward finding significant differences; however, no effect was significant
( $p$ s > .2). Moreover, using the pooled error term from this analysis, separate between-subjects planned comparisons for each stimulus found test session to have no effect $(p s>.39)$. Thus, the following analyses ignored test session and focused on responding to each test stimulus as a function of its training condition. By pooling the data in this manner, in each planned comparison, data from the first session were perfectly balanced (in terms of independent variables, aside from those in the comparison) by data from the second test session. Because the experiment did not use a completely factorial design, these analyses comprised a series of pairwise comparisons, each using its own error term.

The two main analyses were (1) whether responding to $\mathrm{X}$ and $\mathrm{Y}$ was affected by their respective Phase 2 treatments and (2) whether the apparent decrease in overshadowing resulted from increased responding to the overshadowing cue with additional training, as opposed to decreased responding to the baseline cue. Responding to CS Y, which had been reinforced 4 times, was less if it had been reinforced in compound (BY; $M=1.59 \mathrm{log}$ $\mathrm{sec})$ than if it had been reinforced elementally [Y; $M=$ $\left.2.09 \log \sec ; F(1,21)=10.28, M S_{\mathrm{e}}=0.14, p<.01\right]$. In other words, overshadowing of Y was observed. By contrast, responding to CS X, which had been reinforced 36 times, was not significantly affected by its treatment during acquisition (AX vs. $\mathrm{X}$; mean for $\mathrm{AX}=2.16 \mathrm{log} \mathrm{sec}$, mean for $\mathrm{X}=2.10 \log \mathrm{sec} ; p>.70$ ). In other words, overshadowing of $\mathrm{X}$ was not observed. In the compound training condition, responding to CS $\mathrm{X}$ was higher than that to CS Y $\left[F(1,20)=9.59, M S_{\mathrm{e}}=0.19\right]$. CSs X and Y did not differ when trained elementally $(p>.90)$. In other words, the dissipation of overshadowing occurred because responding to the overshadowing cues increased with training, and not because of changes in the elementally trained cues. Another comparison indicated that responding elicited by the overshadowing cues alone, A and $\mathrm{B}$, was not reliably affected by their being reinforced 4 or 36 times, respectively ( $p>.56$; mean for $\mathrm{A}=2.23 \mathrm{log}$ sec; mean for $\mathrm{B}=2.12 \log \mathrm{sec})$.

Experiment 1 demonstrated that the overshadowing seen with 4 compound $\rightarrow$ US pairings is eliminated with 36 such pairings. This dissipation of overshadowing was accomplished by an increase in the response potential of the overshadowed stimulus. It was not accompanied by a change in responding to the potentially overshadowing stimulus. Responding to the more salient stimuli (A and B) reached asymptote within four trials. The observed dissipation in overshadowing cannot be attributed to approach toward an experimenter-imposed response ceiling, because no subjects had a response latency that reached the maximum allowable time of $900 \mathrm{sec}$. The observation that overshadowing was a transient phenomenon with respect to number of training trials raises problems for most contemporary models of Pavlovian learning, which anticipate that overshadowing (as well as other cue competition effects) should not wane (or should even increase) with additional compound trials. 
This observation can be accommodated by a model such as that of Mackintosh (1975), which proposes that the less salient stimulus of a compound conditions more slowly than the more salient stimulus but (at least with some parameters) continues to condition to asymptote $(\lambda)$ over additional training trials. It can also be explained if the overshadowing is overcome by secondorder conditioning mediated by the overshadowing cue (Durlach \& Rescorla, 1980).

The finding that overshadowing dissipates with extended CS $\rightarrow$ US pairings equally raises problems for Miller and Matzel's (1988) comparator hypothesis. On a number of occasions, Miller and his colleagues have referenced the linear operator equation of Bush and Mostellar (1951) to supply the rule by which associative strengths are updated with experience. According to this rule, the strength of association between two stimuli increases according to a monotonic, negatively accelerated function that reaches an asymptotic level with repeated pairings of the stimuli. They have also stated that the associative strength of Link 1 (CS-US association) is compared with the product of the associative strengths of Links 2 and 3 (CS-comparator-stimulus and comparator-stimulus-US associations, respectively; see Figure 1), although the precise nature of this comparison has not been delineated. It should be noted that if the individual associative strengths of Links 2 and 3 grow according to a limited, negatively accelerated function, their product must also be limited and resemble a reversed-S shape similar to a logistic function. This means that overshadowing should be weakest early in training and become progressively more pronounced with additional training, regardless of the precise comparison rule (e.g., subtraction or division) and regardless of the rate at which the product of Links 2 and 3 increases. Clearly, Link 3 must increase more rapidly than Link 1, due to the greater salience of the overshadowing than of the overshadowed cue. Less clear is the rate at which Link 2 should increase relative to Links 1 and 3 according to the model. The component stimuli of Link 2 are in better temporal contiguity than the component stimuli of Links 1 and 3 and, thus, might be expected to associate more quickly. However, the element activated by Link 2 (the overshadowing cue) is ordinarily of lower salience than the elements activated by Links 1 and 3 (the US), making prediction concerning the relative rates of increase of Link 1 and the product of Links 2 and 3 ambiguous. Thus, the model is unable to specify whether the overshadowing deficit should be weak from the outset of training and then grow stronger, or whether it should be of full strength early in training. However, it unambiguously predicts that overshadowing should not get weaker with extended training. The present results demonstrated the opposite outcome.

Miller and colleagues, however, have recently elaborated the comparator hypothesis in a manner that might accommodate the present findings. The extended comparator hypothesis (Denniston, Savastano, \& Miller, 2001) proposes that, just as Link 1 has a comparator stimulus, so can Links 2 and 3 each have their own comparator stimulus. In the model's terminology, a secondorder comparator stimulus for the target CS is any stimulus that serves as a comparator stimulus for Link 2 or Link 3 (see Figure 4). Specifically, a comparator stimulus for Link 2 is any stimulus through which the target CS is indirectly associated with any of the target CS's primary comparator stimuli. Similarly, a comparator stimulus for Link 3 is any stimulus through which any of the target CS's primary comparator stimuli is indirectly associated with the US. To the extent that Links 2 and 3 have robust comparator stimuli themselves, their potential to down-modulate responding supported by Link 1 is attenuated, and net responding elicited by the target CS should be increased. As is true of first-order comparator stimuli, second-order comparator stimuli can be discrete (e.g., Denniston, Savastano, Blaisdell, \& Miller, 2003), or they can be contextual (e.g., Blaisdell, Bristol, Gunther, \& Miller, 1998). Importantly, each stimulus other than the target cue and outcome can function both as a first-order comparator stimulus (which may be down modulated by second-order comparator stimuli) and as a second-order comparator stimulus (which may downmodulate other first-order comparator stimuli).

Given a first-order comparator stimulus other than the conditioning context, the conditioning context can become a comparator stimulus for Links 2 and 3 (i.e., a secondorder comparator stimulus for the target CS), thereby down-modulating the potential of a first-order comparator stimulus to down-modulate responding supported by Link 1. For example, Stout, Chang, and Miller (2003) used massed $\mathrm{AX} \rightarrow$ outcome pairings to encourage strong associations between the target CS (X) and the context, the context and the overshadowing CS (A), and the context and the outcome (a US in one experiment, and a surrogate US in another). Consistent with the extended comparator hypothesis, overshadowing was eliminated by massed $\mathrm{AX} \rightarrow$ outcome trials. In contrast, control groups that received widely spaced $\mathrm{AX} \rightarrow$ outcome trials showed the conventional overshadowing deficit. With spaced trials, the conditioning context presumably played little role as a second-order comparator stimulus for the overshadowed cue (first-order for the overshadowing cue), due to the large amount of extinction of the context-CS association during the long intertrial intervals. Further evidence for a role of the conditioning context as a secondorder comparator stimulus for the target CS was provided by the observation that subsequent extinction of the conditioning context following massed $\mathrm{AX} \rightarrow$ outcome pairings reduced responding to the target stimulus (i.e., increased overshadowing), whereas the same treatment following spaced AX $\rightarrow$ outcome pairings tended to increase responding to the target stimulus (i.e., reduce overshadowing). The extended comparator hypothesis explains the effect of posttraining context extinction following massed training by assuming that extinction treatment reduces the context's potential to serve as a second-order comparator stimulus for Links 2 and 3. Following con- 

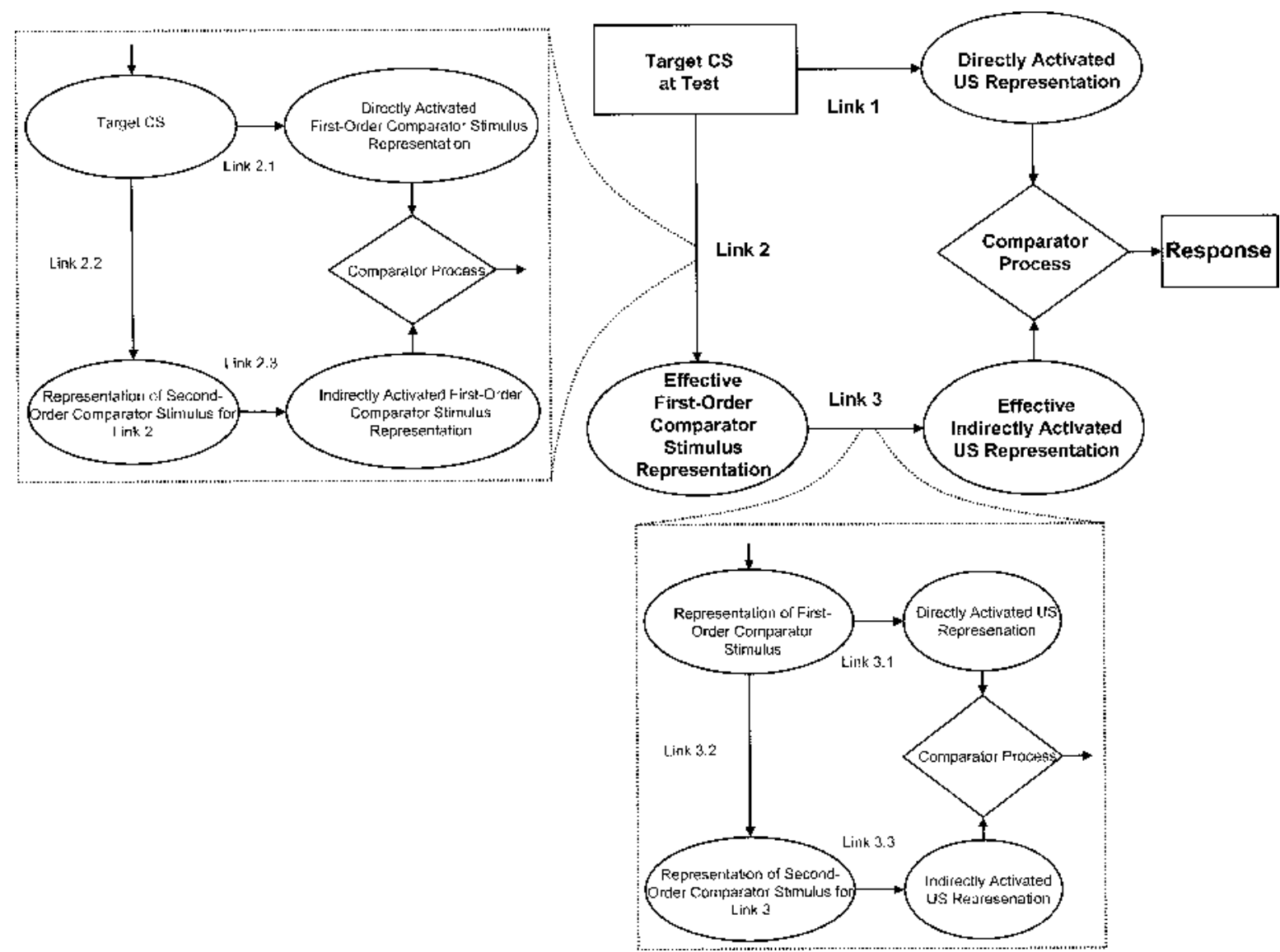

Figure 4. The extended comparator hy pothesis (Denniston, Savastano, \& Miller, 2001). Applied to extended training with the $A X$ compound in the present research, the target conditioned stimulus (CS) is $X, A$ is the effective first-order comparator stimulus, the context is the effective second-order comparator stimulus for both Link 2 and Link 3, and the unconditioned stimulus (US) is the footshock.

text extinction, the overshadowing stimulus is no longer attenuated in its potential to serve as a first-order comparator stimulus, and consequently, responding to the target stimulus declines. In contrast, posttraining extinction of the training context following spaced AX $\rightarrow$ outcome acquisition training had little effect, because the context already underwent appreciable extinction during the long intertrial intervals of training. For another example of the conditioning context's serving as a secondorder comparator stimulus for the target stimulus, see Blaisdell, Bristol, et al. (1998).

How, then, might the extended comparator hypothesis explain the dissipation in overshadowing seen with extended overshadowing training in the present research? That is, given that the conditioning context can apparently function as a second-order comparator stimulus for a target stimulus that is trained in compound (i.e., overshadowing), why would it be more likely to serve as an effective second-order comparator stimulus following extended reinforcement? A possible answer lies in different dynamic changes in the effective strength of
Link 1 relative to the first- and second-order comparator processes. The overall strength of the second-order process depends on the growth of the function governing down-modulation of Link 2 multiplied by the function governing down-modulation of Link 3 . The function down-modulating Link 2 is the product of the $\mathrm{X}$-context association and the context-A association (see Figure 4). The function modulating Link 3 is the product of the A-context association and the context-US association. Presumably, each of these component associations with the context should grow over trials in a negatively accelerated manner. Because the context is of low salience and stands in poor temporal relationship to the stimulus components of the second-order comparator links (i.e., the target cue, overshadowing stimulus, and US), each of these components of the second-order process should grow at a rate that is slower than Links 1,2, or 3. Hence, the function describing the growth of the second-order comparator process over consistently reinforced compound conditioning trials should, like the function describing the growth of the first-order comparator process 


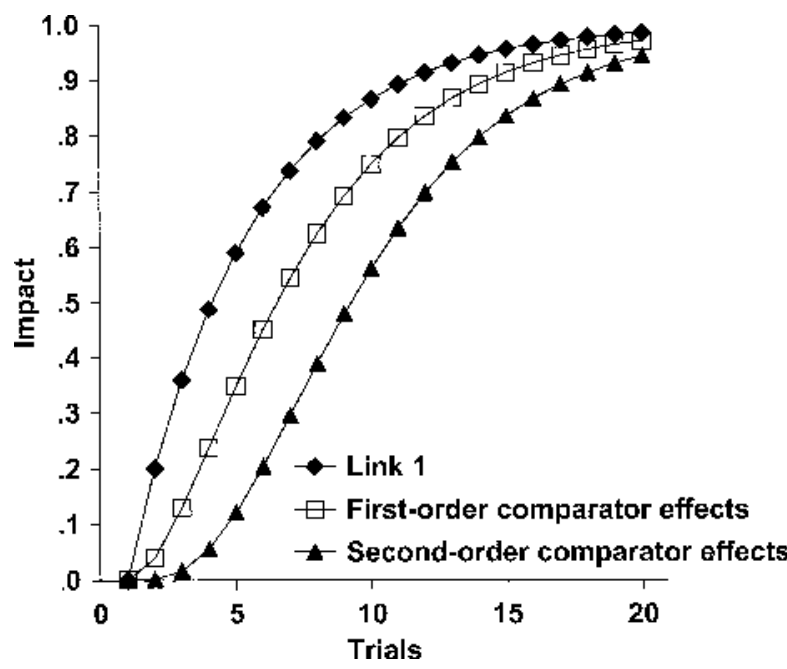

Figure 5. Hypothetical time course of the impact of the response-modulating functions of the extended comparator hypothesis. Link 1 supports conditioned responding. The firstorder comparator process attenuates it. The second-order comparator process attenuates the attenuating potential of the first-order process. The rate at which the product of Links 2 and 3 grows is not determined by the model (see the text).

(i.e., the product of Links 2 and 3), resemble a reversed$\mathrm{S}$ shape, but because it is the product of slower growing component associations, it should ascend more slowly. Thus, although the extended comparator hypothesis is silent as to the mathematical function by which the directly and the indirectly activated US representations are compared, it does make a clear prediction regarding the relative course of overshadowing as a function of number of trials concerning the strengths of the responsefacilitating comparator links for Links 2 and 3 relative to the other comparator processes (see Figure 5). These considerations lead to the prediction that overt behavior as a function of number of trials should eventually increase (i.e., overshadowing should decrease) with extended acquisition training. (Third-order and higher comparator processes are ignored here because a damping factor is assumed to make weaker each successive order of comparator processes.) Put simply, we are proposing that following extended training, the conditioning context overshadows the overshadowing cue.
The account that we are proposing can be differentiated from that proposed by Mackintosh (1975), which also views overshadowing as potentially reversible through extended compound training trials, in that our account uniquely predicts that extinction of the acquisition context following the dissipation of overshadowing should restore the overshadowing deficit. Our account also can be differentiated from the notion that overshadowing dissipates because of second-order conditioning through the overshadowed cue, because this view does not anticipate any effect of context extinction.

\section{EXPERIMENT 2}

In Experiment 2, we tested the possibility that the dissipation of overshadowing observed in Experiment 1 was due to the conditioning context's becoming an effective second-order comparator stimulus for the target stimulus due to the extended pairings between the CS compound and the outcome. If this is true, massive extinction of the context prior to testing of CS X should increase CS A's potential to serve as a first-order comparator stimulus and, therefore, increase overshadowing of CS X.

Previous experimental analyses indicate that reducing excitatory responding in nonhuman subjects through posttraining manipulation of the comparator stimulus is more easily observed in a sensory preconditioning paradigm in which the outcome of the target association is a stimulus of low biological significance that only is later made behaviorally potent by its being paired with a US (e.g., Denniston, Miller, \& Matute, 1996). The protective effect of biological significance helps explain why a reduction of responding was not observed (as predicted by the comparator hypothesis) following overtraining of the elementally reinforced cue. Toward obtaining a good animal model of the chronicled human sensitivity to posttraining deflation of associates of a target stimulus (e.g., Wasserman \& Berglan, 1998), we switched from the first-order conditioning of Experiment 1 to sensory preconditioning in Experiment 2. Another advantage of this switch was to examine the generality of the dissipationof-overshadowing effect observed in Experiment 1 in a somewhat different preparation. To reduce the number of groups required for the experiment, the experimental design confounded the overshadowing treatments of CS X and CS Y. That is, treatment of X (X and AX) was

Table 2

Experiment 2 Design Summary

\begin{tabular}{lllccc}
\hline \multicolumn{1}{c}{ Group } & \multicolumn{1}{c}{ Ph 1 in Cxt2 } & Ph 2 in Cxt2 & Ph 3 in Cxt2 & Ph 4 Cxt1 & Test in Cxt1 \\
\hline Comp/Cntl & A-, B-, X-, Y- & $\mathrm{AX} \rightarrow \mathrm{S}, \mathrm{BY} \rightarrow \mathrm{S}$ & Short Cxt & $\mathrm{S} \rightarrow \mathrm{US}$ & $\mathrm{X}, \mathrm{Y}$ \\
Comp/Cxt- & $\mathrm{A}-, \mathrm{B}-, \mathrm{X}-, \mathrm{Y}-$ & $\mathrm{AX} \rightarrow \mathrm{S}, \mathrm{BY} \rightarrow \mathrm{S}$ & Long Cxt & $\mathrm{S} \rightarrow \mathrm{US}$ & $\mathrm{X}, \mathrm{Y}$ \\
Ele/Cntl & $\mathrm{A}-, \mathrm{B}-, \mathrm{X}-, \mathrm{Y}-$ & $\mathrm{X} \rightarrow \mathrm{S}, \mathrm{Y} \rightarrow \mathrm{S}$ & Short Cxt & $\mathrm{S} \rightarrow \mathrm{US}$ & $\mathrm{X}, \mathrm{Y}$ \\
Ele/Cxt- & $\mathrm{A}-, \mathrm{B}-, \mathrm{X}-, \mathrm{Y}-$ & $\mathrm{X} \rightarrow \mathrm{S}, \mathrm{Y} \rightarrow \mathrm{S}$ & Long Cxt & $\mathrm{S} \rightarrow \mathrm{US}$ & $\mathrm{X}, \mathrm{Y}$ \\
\hline
\end{tabular}

Notes-Comp, compound; Ele, element; Cntl, short duration context exposure; $\mathrm{Cxt}-$, long duration context exposure; Cxt1, Context 1; Cxt2, Context 2; X, low salience target stimulus presented with the outcome 36 times in Phase 2; Y, low salience target stimulus presented with the outcome 4 times in Phase 2; A, high salience (overshadowing) stimulus for X; B, high salience (overshadowing) stimulus for Y; S, surrogate US; US, footshock; -, no outcome trial; $\rightarrow$, followed by. 
not counterbalanced against treatment of Y ( $\mathrm{Y}$ and B Y; compare Table 2 with Table 1). This strategy seemed reasonable because Experiment 1 showed that treatment of each target stimulus had no significant effect on responding to the other stimulus.

\section{Method}

\section{Subjects and Apparatus}

The apparatus was the same as that described in Experiment 1. The subjects were 24 male and 24 female Sprague Dawley rats, bred in our colony. Housing and deprivation conditions were the same as those described in Experiment 1 . The subjects were assigned to four groups of $n=12$ (see Table 2): Comp/Cntl, Comp/Cxt-, Ele/Cntl, Ele/Cxt-. The first term in the group name designates the nature of the acquisition phase (Phase 2; Comp = compound stimulus, Ele $=$ elemental stimulus); the second term designates the duration of context extinction subsequently administered (Phase 3 ). Subjects in the $\mathrm{Cxt}-$ condition received $10 \mathrm{~h}$ of nonreinforced exposure to the experimental context, whereas those in the Cntl condition received $20 \mathrm{~min}$ of nonreinforced exposure to the experimental context. Previous experimental analyses from our laboratory indicate that the amount of context time administered to the subjects in the Cntl condition was much less than the amount necessary to produce comparator devaluation effects.

\section{Procedure (see Table 2)}

Acclimation. The 60-min acclimation session on Day 1 was identical to that in Experiment 1 and occurred in Context 1.

Phase 1 (stimulus preexposure). On Day 2, the four conditioning stimuli were presented to all the animals in Context 2, following the same procedure as that described in Experiment 1. Stimulus preexposure was included to equate the subject's experience with that of the subjects in Experiment 1, thereby facilitating the external validity of possible cross-experiment comparisons.

Phase 2 (training). Phase 2 spanned Days 3-12 and consisted of an identical number and temporal distribution of $\mathrm{X}$ (36 trials) and $\mathrm{Y}$ (4 trials) trials, as in Phase 2 of Experiment 1. A change from the treatment in Experiment 1 involved the use of a flashing light as a surrogate US (S) in place of footshock. The flashing light began $3 \mathrm{sec}$ prior to the termination of the CS, thereby coterminating with the $\mathrm{X}$ or Y stimulus. As in Experiment 1, the experimental context of treatment during Phase 2 (Context 2) was different from that used for acclimation, reacclimation, and testing (Context 1). Session durations were the same as those in Experiment 1. The subjects received one of two treatments. Either CSs X and Y were reinforced alone (Ele), or they were reinforced in compound (Comp) with CSs A and B, respectively (see Table 2). As in Experiment $1, \mathrm{X}$ and $\mathrm{Y}$ were counterbalanced, as were A and B. Lick tubes were not available.

Phase 3 (context extinction). On Days 13-16, half of the subjects received long- and half short-duration exposure to Context 2 with no nominal stimulus presentations and with the lick tubes removed. Context extinction ( $\mathrm{Cxt}-$ ) consisted of daily exposure to the context for $2.5 \mathrm{~h}$. Every $50 \mathrm{~min}$, the sound-attenuating enclosures were opened, and the conditioning chambers were briefly shaken, to ensure that the animals were awake. The control (Cntl) treatment consisted of daily exposure to the context for $5 \mathrm{~min}$; this controlled for any possible effects of handling on long context extinction subjects.

Phase 4 (surrogate inflation). On Day 17, all the subjects received four pairings per session of the Stimulus $\mathrm{S}$ (the flashing light) and the footshock during a 1-h session in Context 1. Footshocks were presented at $15,21,36$, and 52 min into the session. Stimulus S was presented $2.5 \mathrm{sec}$ prior to the onset of the 0.5 -sec footshock, with $\mathrm{S}$ and the footshock being simultaneously terminated. No lick tubes were present.

Reacclimation. On Days 18 and 19, all the subjects were given one daily exposure to Context 1 with lick tubes available. No nom- inal stimuli were presented. The latency to complete the first five cumulative seconds of licking was recorded during each 60-min session. On the second reacclimation session, all the subjects readily drank within 60 -sec of placement into the testing context.

Testing. On Days 20 and 21, fear of the test stimulus was measured with the same procedure as that described in Experiment 1. The target CSs, $\mathrm{X}$ and $\mathrm{Y}$, were tested in separate sessions, one per day, with the order of testing counterbalanced within groups.

\section{RESULTS AND DISCUSSION}

The results of Experiment 2 are presented in Figure 6. As can be seen, the observation in Experiment 1 that overshadowing dissipates with extended training was replicated in the groups that received short exposure to the conditioning context (Comp vs. Ele in the control condition). Responding elicited by CS Y after four compound training trials was less than that elicited by its elementally reinforced control. However, responding elicited by CS X, which was reinforced 36 times, displayed the opposite (nonsignificant) tendency. Importantly, as predicted by the extended comparator hypothesis, massive extinction of the experimental context (Condition Cxt-) reduced responding elicited by both overshadowed cues. As a result, the overshadowing effect was now observed with both cues (Group Comp/Cxt- vs. Ele/Cxt-). Statistical analyses confirm these conclusions.

Due to pre-CS times greater than $60 \mathrm{sec}$ on at least 1 of the 2 test days, 1 subject in Group Ele/Short and 1 subject in Group Ele/Long were eliminated from the study. Pre-CS times were first analyzed with a training stimulus $($ Comp vs. Ele $) \times$ context treatment $\left(\right.$ Cntl vs. $\left.\mathrm{Cxt}^{-}\right) \times$ test stimulus (X vs. Y) mixed-model ANOVA. The main effects and interactions were nonsignificant $(p>.17)$, indicating similar baseline suppression times between groups.

CS times were analyzed with a training condition $($ Comp vs. Ele $) \times$ context treatment (short vs. long $) \times$ test stimulus (X vs. Y) mixed-model ANOVA. The three main effects were significant [training condition, $F(1,42)=$ $26.95, M S_{\mathrm{e}}=0.25, p<.001 ;$ context extinction, $F(1,42)=$ $6.71, M S_{\mathrm{e}}=0.25, p<.02$; test stimulus, $F(1,42)=5.65$, $\left.M S_{\mathrm{e}}=0.12, p<.03\right]$. Duration of context extinction interacted with training condition $\left[F(1,42)=21.53, M S_{\mathrm{e}}=\right.$ $0.25, p<.001]$. The triple interaction was also significant $\left[F(1,42)=7.89, M S_{\mathrm{e}}=0.12, p<.01\right]$. No other interactions reached statistical significance $(p \mathrm{~s}>.05)$.

More illuminating were a series of planned comparisons and simple interactions. Comparisons restricted to the subjects that received short context exposure revealed that overshadowing dissipated with extended CS-US pairings, thereby replicating our finding in Experiment 1 . That is, the overshadowing effect seen with CS Y (presented 4 times) was appreciable, with suppression to CS Y being less after BY-US training $(M=$ $1.35 \log \mathrm{sec}$ ) than after Y-US training [ $M=1.74 \log \mathrm{sec}$; $\left.F(1,42)=4.64, M S_{\mathrm{e}}=0.19, p<.05\right]$, whereas there was a nonsignificant tendency toward reversal (i.e., potentiation) seen with CS X (presented 36 times), with suppression to CS X tending to be greater after AX-US 


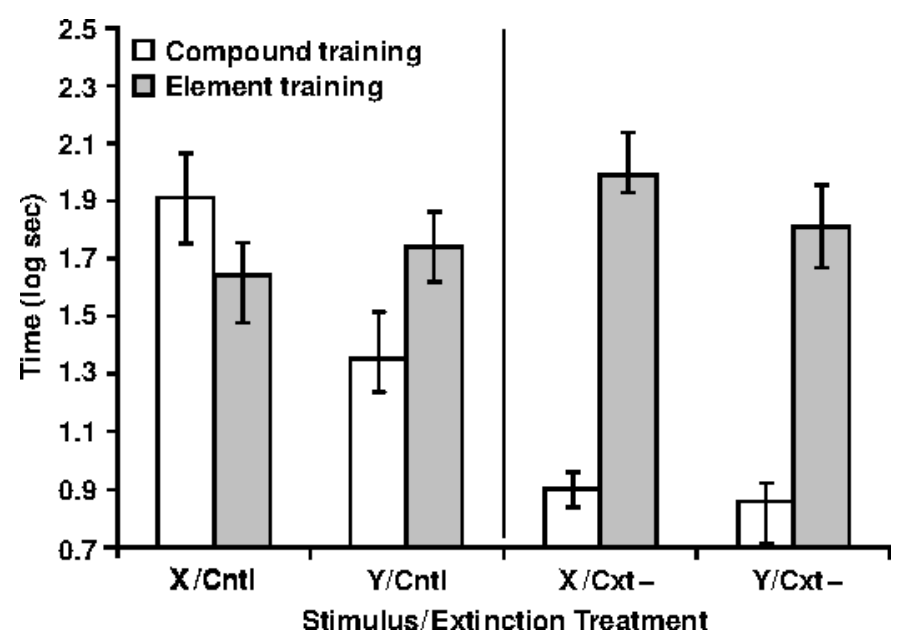

\begin{abstract}
Figure 6. Mean times to resume drinking upon presentation of target conditioned stimuli (CSs) that had been paired with an innocuous stimulus (S) later made potent through pairings with a shock unconditioned stimulus in Experiment 2. CS X was reinforced 36 times, and CS Y was reinforced 4 times. Both $X$ and $Y$ were paired with $S$, either alone or in compound with a more salient stimulus (A or B). Half of the subjects then received $10 \mathrm{~h}$ of exposure to the training context $(\mathrm{Cxt}-)$, and half received 20 min of exposure to the training context (Cntl). At test, the stimuli were presented alone for all the subjects. Error bars indicate standard errors of the means.
\end{abstract}

training $(M=1.91 \log \mathrm{sec})$ than after $\mathrm{X}-\mathrm{US}$ training $[M=$ $\left.1.64 \log \sec ; F(1,42)=2.41, M S_{\mathrm{e}}=0.18, p>.12\right]$. These findings are consistent with a significant interaction of training condition and test stimulus restricted to the short condition $\left[F(1,12)=6.25, M S_{\mathrm{e}}=0.18, p<.05\right]$. Moreover, overshadowing dissipated in a manner similar to that in Experiment 1, with responding to CS X trained in compound greater than responding to $\mathrm{CS} \mathrm{Y}$ trained in compound $\left[F(1,11)=7.28, M S_{\mathrm{e}}=0.26, p<.05\right]$, but with responding to both cues trained elementally not differing significantly $(p>.67)$.

Subjects that received the long duration of context exposure demonstrated an overshadowing effect with both stimuli. Thus, for CS Y, responding was less following compound training $(M=0.86 \log \mathrm{sec})$ than following element training $\left[M=1.81 \log \sec ; F(1,42)=27.64, M S_{\mathrm{e}}=\right.$ $0.19, p<.001]$, and for CS X, responding was similarly less following compound training $(M=0.90 \mathrm{log} \mathrm{sec})$ than following element training $[M=1.99 \mathrm{log} \mathrm{sec}$; $\left.F(1,42)=38.28, M S_{\mathrm{e}}=0.18, p<.001\right]$. That the overshadowing effect was comparable for both CSs X and Y was confirmed by a nonsignificant interaction between training condition and test stimulus $(p>.34)$. Another way to confirm the visual pattern of Figure 6 is to examine whether context extinction interacted with training condition for both cues. It did, for CS X $[F(1,43)=$ $\left.25.69, M S_{\mathrm{e}}=0.19, p<.001\right]$ and for CS Y $[F(1,44)=$ $\left.5.87, M S_{\mathrm{e}}=0.19, p<.05\right]$. This observation is consistent with the reversal from the nonsignificant potentiation ef- fect to overshadowing seen with CS $\mathrm{X}$ as a function of context extinction and with the increased size of the overshadowing effect seen to CS Y as a function of context extinction. Finally, contrary to the prediction of the extended comparator hypothesis, context extinction did not significantly enhance responding to either elementally trained test cue ( $p$ s > .16), although the tendencies were in the predicted direction. Thus, statistical analyses confirmed that overshadowing dissipated with extensive training in a sensory preconditioning paradigm and that overshadowing was restored by massive extinction of the training context. These results were generally consistent with the predictions of the extended comparator hypothesis. The reader may wonder why the surrogate stimulus does not act as a comparator stimulus. The simple answer is that we do not know. Neither the comparator hypothesis nor the extended comparator hypothesis is able to account for sensory preconditioning or second-order conditioning. In each of these procedures, a cue is linked to the US only indirectly, yet excitatory responding is observed. Clearly, under certain conditions, indirect associative links between a cue and a US can transmit excitation rather than play an opposing (comparator) role. We are currently developing a model intended to delimit the conditions under which the comparator process will become engaged. Here, we simply must acknowledge that positive mediation between the target cue and the US through the surrogate US is incompatible with the extended comparator hypothesis in its present form. 


\section{GENERAL DISCUSSION}

In the present experiments, we examined the effect of extended acquisition trials on the magnitude of the overshadowing deficit. Experiment 1 demonstrated that the overshadowing effect waned as the number of CS-US pairings was increased from 4 to 36 . This increased responding to the overshadowed stimulus could not be attributed to differences in the number of shocks between subjects. Moreover, it was not accompanied by a concomitant rise in conditioned responding elicited by the overshadowing stimuli. The latter observation is consistent with the interpretation that the overshadowing treatment did not change the asymptote of responding supportable by the US but, instead, slowed the approach of conditioned responding elicited by the less salient stimulus toward an asymptote that was common with that of the more salient stimulus (Mackintosh, 1975). The possibility of an experimenter-imposed response ceiling's capping conditioned responding elicited by CSs A, B, and $\mathrm{X}$ at the same level (and thus producing an artifactual asymptote) was precluded by the observation that none of the subject's suppression times equaled the ceiling time imposed during the testing procedure.

Experiment 2 replicated the dissipation-of-overshadowing effect, using a sensory preconditioning paradigm, suggesting that the effect has some generality and does not require a large number of footshocks to be observed. Moreover, massive extinction of the acquisition context reduced responding to both of the less salient stimuli that had been reinforced in compound ( $\mathrm{X}$ and $\mathrm{Y}$ ), having the largest decrementing effect on responding elicited by the stimulus that had been reinforced in compound 36 times (X). That is, the overshadowing effect eliminated by extended training was restored by massive extinction of the experimental context. According to the extended comparator hypothesis (Denniston et al., 2001), extensive $\mathrm{AX} \rightarrow$ US pairings cause the conditioning context (as a second-order comparator stimulus for target CS X) to down-modulate the overshadowing stimulus's potential to down-modulate responding to target CS X. This prediction stems from second-order comparator effects' slowly increasing to their asymptote, as indicated in Figure 5 (see the Results and Discussion section of Experiment 1). Because this waning of overshadowing with extended training depends on the associative status of the second-order comparator stimulus at the time of testing, it should be reversible through postacquisition extinction of the effective second-order comparator stimulus, which was the context in this case. In contrast, Mackintosh's (1975) theory of selective attention interprets this dissipation-of-overshadowing effect as slowed acquisition of the association between the overshadowed CS and the US and, thus, does not clearly predict an effect of postacquisition extinction of the acquisition context. The within-compound view, according to which CS A becomes a second-order excitor that attenuates the overshadowing effect, does not predict the effect of context extinction in restoring overshadowing. Of contemporary learning models, only the extended comparator hypothesis makes a prediction about the effect of contextual extinction in this situation.

Nonetheless, the extended comparator hypothesis's account of the present data is not without its failings. For example, the model loosely predicts that in the element conditions, responding should be greater to CS Y than to CS X, since the potential of the context to serve as a first-order comparator stimulus should increase with additionalCS-US pairings. No such trend was observed in either experiment, including Experiment 2, which was conducted with a sensory preconditioning procedure that has been shown to be sensitive to decreases in responding produced by manipulations, such as contextual inflation, that should increase the strength of the products of Links 2 and 3. As was noted with respect to basic overshadowing, the prediction of reduced responding following overtraining is not clear-cut, since the training context as a first-order comparator might have reached asymptotic strength within four CS-US pairings and, therefore, would not have been detectable given the present training parameters. However, this possibility is not consistent with the lack of any observed effect of extinction of the conditioning context on responding to CS X or Y following element training in Experiment 2.

Of course, there are alternatives to the extended comparator hypothesis's explanation of the results. One possibility is that the CS that has been reinforced in compound with CS A 36 times is not overshadowed because of second-order conditioning through the conditioning context. This explanation is consistent with the fact that the context extinction manipulation of Experiment 2 reduced responding to CS X. However, a problem with this explanation lies in the high responding following context extinction elicited by the $\mathrm{X}$ cue trained elementally, which would seem to rule out any second-order conditioning in this condition. Thus, for this account to work, one must assume that the overshadowing treatment interfered with the acquisition of the X-US association, leaving a lower level of responding to be revealed after the $\mathrm{X}$-context-US association was extinguished. However, the nearly complete recovery of overshadowing that is often observed when the overshadowing cue is extinguished (e.g., Kaufman \& Bolles, 1981; but admittedly not tested here) would seem to rule out the possibility that the cue-outcome association is affected to any great degree by the overshadowing treatment.

In conclusion, the present experiments provide the first well-controlled demonstration that overshadowing wanes with a sufficient number of compound stimulus training trials. They further suggest that this decline in overshadowing depends on overshadowing of the discrete overshadowing cue by the conditioning context, since posttraining extinction of the training context was observed to restore overshadowing following many compound training trials. In conjunction with Azorlosa and Cicala's (1988) demonstration of the waning of 
blocking with extended compound trials, the present experiment's demonstration of the dissipation in overshadowing following extended training suggests that conditioning theorists might more profitably view these forms of cue competition effects as deficits in the rate of acquisition of responding to the cue subjected to competition, rather than as deficits in that cue's asymptotic performance level, as is now often assumed. At present, these observations appear to be most compatible with Denniston et al.'s (2001) extended comparator hypothesis, with the additional assumption that first- and secondorder comparator processes become engaged on dissociable time scales.

\section{REFERENCES}

Azorlosa, J. L., \& Cicala, G. A. (1988). Increased conditioning in rats to a blocked CS after the first compound trial. Bulletin of the Psychonomic Society, 26, 254-257.

Bellingham, W. P., \& Gillette, K. (1981). Attenuation of overshadowing as a function of nondifferential compound conditioning trials. Bulletin of the Psychonomic Society, 18, 218-220.

Blaisdell, A. P., Bristol, A. S., Gunther, L. M., \& Miller, R. R. (1998). Overshadowing and latent inhibition counteract each other: Support for the comparator hypothesis. Journal of Experimental Psychology: Animal Behavior Processes, 24, 335-351.

Blaisdell, A. P., Denniston, J. C., \& Miller, R. R. (1998). Temporal encoding as a determinant of overshadowing. Journal of Experimental Psychology: Animal Behavior Processes, 24, 72-83.

Brandon, S. E., Vogel, E. H., \& Wagner, A. R. (2000). A componential view of configural cues in generalization and discrimination in Pavlovian conditioning. Behavioural Brain Research, 110, 67-72.

Bush, R. R., \& Mostellar, F. (1951). A mathematical model for simple learning. Psychological Review, 58, 313-323.

Cole, R. P., Oberling, P., \& Miller, R. R. (1999). Recovery from onetrial overshadowing. Psychonomic Bulletin \& Review, 6, 424-431.

Denniston, J. C., Miller, R. R., \& Matute, H. (1996). Biological relevance as a determinant of cue competition. Psychological Science, 7, 325-331.

Denniston, J. C., Savastano, H. I., Blaisdell, A. P., \& Miller, R. R. (2003). Cue competition as a retrieval deficit. Learning \& Motivation, 34, 1-31.

Denniston, J. C., Savastano, H. I., \& Miller, R. R. (2001). The extended comparator hypothesis: Learning by contiguity, responding by relative strength. In R. Mowrer \& S. Klein (Eds.), Handbook of contemporarylearning theories (pp. 65-117). Hillsdale, NJ: Erlbaum.

Durlach, P. J., \& Rescorla, R. A. (1980). Potentiation rather than overshadowing in flavor-aversion learning: An analysis in terms of within-compound associations. Journal of Experimental Psychology: Animal Behavior Processes, 6, 175-187.

Gallistel, R., \& Gibbon, J. (2000). Time, rate, and conditioning.Psychological Review, 107, 289-344.

Holland, P. C. (1999). Overshadowing and blocking as acquisition deficits: No recovery after extinction of overshadowing or blocking cues. Quarterly Journal of Experimental Psychology, 52B, 307-334.

KAMIN, L. J. (1969). Predictability, surprise, attention, and conditioning. In B. A. Campbell \& R. M. Church (Eds.), Punishment and aversive behavior (pp. 279-296). New York: Appleton-Century-Crofts.

Kaufman, M. A., \& Bolles, R. C. (1981). A nonassociative aspect of overshadowing. Bulletin of the Psychonomic Society, 18, 318-320.
Kehoe, E. J. (1982). Overshadowing and summation in compound stimulus conditioning of the rabbit's nictitating membrane response. Journal of Experimental Psychology: Animal Behavior Processes, $\mathbf{8}$, 313-328.

Mackintosh, N. J. (1975). A theory of attention: Variations in the associability of stimuli with reinforcement. Psychological Review, 82, 276-298.

Matzel, L. D., Schachtman, T. R., \& Miller, R. R. (1985). Recovery of an overshadowed association achieved by extinction of the overshadowing stimulus. Learning \& Motivation, 16, 398-412.

McLaren, I. P. L., \& Mackintosh, N. J. (2000). An elemental model of associative learning: I. Latent inhibition and perceptual learning. Animal Learning \& Behavior, 28, 211-246.

Miller, R. R., Barnet, R. C., \& Grahame, N. J. (1992). Responding to a conditioned stimulus depends on the current associative status of other cues that were present during training of that specific stimulus. Journal of Experimental Psychology: Animal Behavior Processes, 18, 251-264.

Miller, R. R., \& Escobar, M. (2002). Learning: Laws and models of basic conditioning. In C. R. Gallistel (Ed.), Stevens' handbook of experimental psychology: Vol 3. Learning, motivation, and emotion (3rd ed., pp. 47-102). New York: Wiley.

Miller, R. R., \& MATZel, L. D. (1988). The comparator hypothesis: A response rule for the expression of associations. In G. H. Bower (Ed.), The psychology of learning and motivation (Vol. 22, pp. 1-92). Orlando, FL: Academic Press.

Pavlov, I. P. (1927). Conditioned reflexes (G. V. Anrep, Trans.). London: Oxford University Press.

Pearce, J. M. (1987). A model of stimulus generalization for Pavlovian conditioning. Psychological Review, 94, 61-73.

Pearce, J. M., \& Hall, G. (1980). A model for Pavlovian learning: Variations in the effectiveness of conditioned but not unconditioned stimuli. Psychological Review, 82, 532-552.

Price, P. C., \& YATES, J. F. (1993). Judgmental overshadowing: Further evidence of cue interaction in contingency judgment. Memory \& Cognition, 21, 561-572.

Rauhut, A. S., McPhee, J. E., \& Ayres, J. J. B. (1998). Blocked and overshadowed stimuli are weakened in their ability to serve as blockers and second-order reinforcers in Pavlovian fear conditioning. Journal of Experimental Psychology: Animal Behavior Processes, 25, 45-67.

Rescorla, R. A., \& Wagner, A. R. (1972). A theory of Pavlovian conditioning: Variations in the effectiveness of reinforcement and nonreinforcement. In A. H. Black \& W. F. Prokasy (Eds.), Classical conditioning II: Current research and theory (pp. 64-99). New York: Appleton-Century-Crofts.

Rusiniak, K. W., Hankins, W. G., Garcia, J., \& Brett, L. P. (1979). Flavor-illness aversions: Potentiation of odor by taste in rats. Behavioral \& Neural Biology, 25, 1-17.

Stout, S. C., Chang, R., \& Miller, R. R. (2003). Trial spacing as a determinant of cue interaction. Journal of Experimental Psychology: Animal Behavior Processes, 29, 23-38.

Van Hamme, L. J., \& Wasserman, E. A. (1994). Cue competition in causality judgments: The role of nonpresentation of compound stimulus elements. Learning \& Motivation, 25, 127-151.

Wasserman, E. A., \& Berglan, L. R. (1998). Backward blocking and recovery from overshadowing in human causal judgement: The role of within-compound associations. Quarterly Journal of Experimental Psychology, 51B, 121-128.

(Manuscript received June 17, 2002; revision accepted for publication January 6, 2003.) 